Prepared for the U.S. Department of Energy

under Contract DE-AC05-76RL01830

\title{
Acoustic Modeling for Aqua Ventus I off Monhegan Island, ME
}

Jonathan Whiting

Luke Hanna

Nicole Dechello

Andrea Copping

October 2013

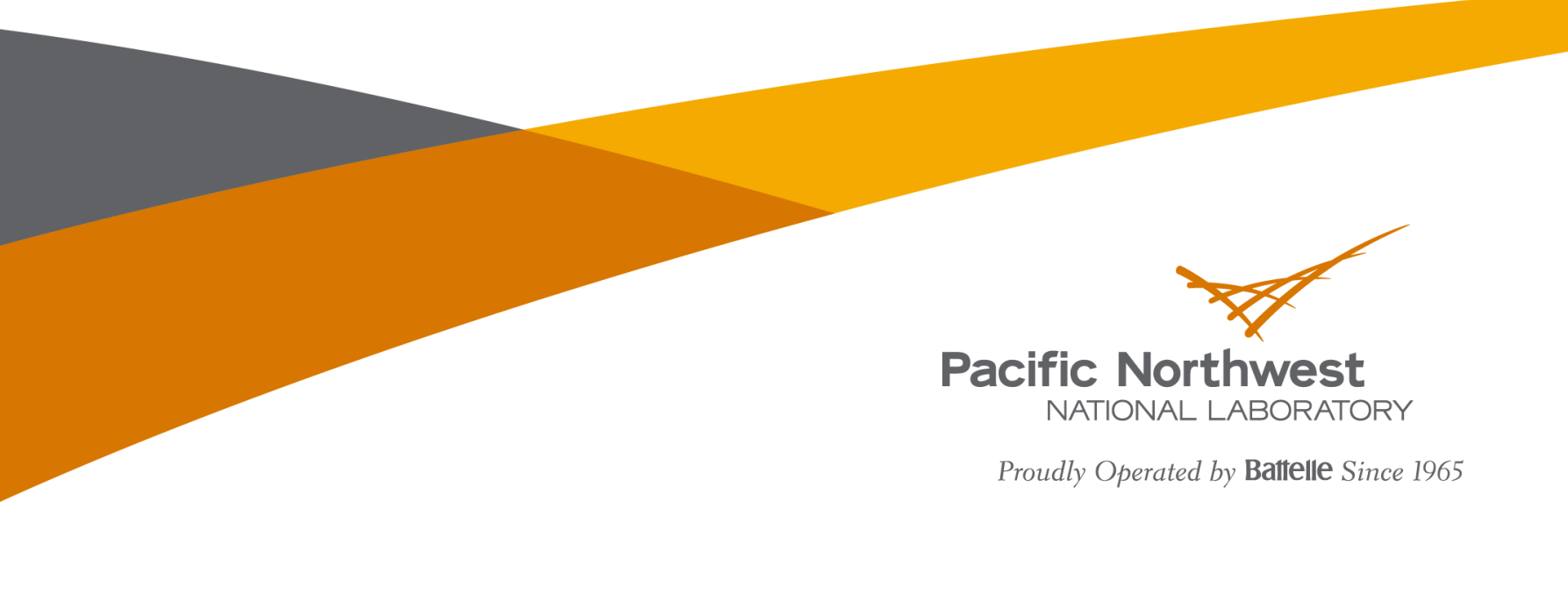




\title{
DISCI.AIMER
}

This report was prepared as an account of work sponsored by an agency of the United States (rovernment. Neither the United States Government nor any agency thereof, nor Battelle Memorial Institute, nor any of their employees, makes any warranty, express or implied, or assumes any legal liability or responsibility for the accuracy, completeness, or usefulness of any information, apparatus, product, or process disclosed, or represents that its use would not infringe privately owned rights. Reference herein to any specific commercial product, process, or service by trade name, trademark, manufacturer, or otherwise does not necessarily constitute or imply its endorsement, recommendation, or favoring by the United States Government or any agency thereof, or Battelle Memorial Institute. The views and opinions of authors expressed herein do not necessarily state or reflect those of the United States Government or any agency thereof.

\author{
PACIFIC NORIHWEST NATIONAL LABORATORY \\ operated by \\ BATTELLE \\ for the \\ UNITED STATES DEPARTMENT OF ENERGY \\ under Contract DE-ACO5-76RL01830
}

Printed in the United States of America

$\Lambda$ vailable to DOE and DOE contractors from the

Office of Scientific and Technical Information,

P.O. Box 62, Oak Ridge, TN 37831-0062;

ph: (865) 576-8401

fax: (865) 576-5728

email: reports $(\bar{a})$ adonis.osti.gov

\footnotetext{
Available to the public from the National Technical Information Service,

U.S. Department of Commerce, 5285 Port Royal Rd., Springfield, VA 22161

ph: (800) 553-6847

fax: $(703) 605-6900$

email: orders@ntis.fedworld.gov

online ordering: http://www.ntis.gov/ordering.htm
}

This document was printed on recycled paper. 
PNNL - 22926

\title{
Acoustic Modeling for Aqua Ventus I off Monhegan Island, ME
}

\author{
Jonathan Whiting \\ Luke Hanna \\ Nicole Dechello \\ Andrea Copping
}

October 2013

Prepared for

the U.S. Department of Energy

under Contract DE-AC05-76RL01830

Pacific Northwest National Laboratory

Seattle Washington 98109 
PNNL - 22926

\section{Table of Contents}

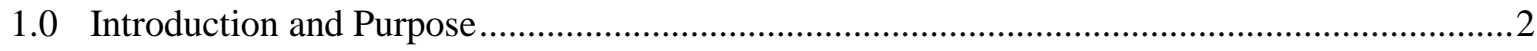

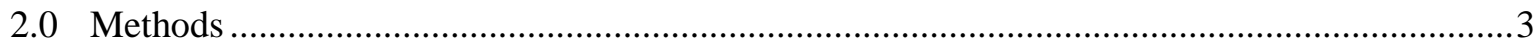

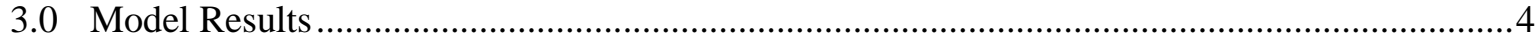

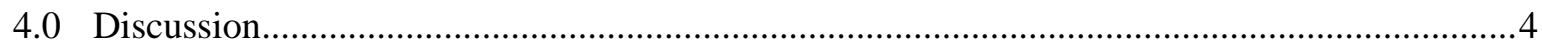

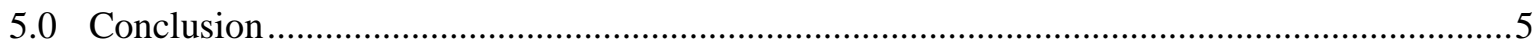

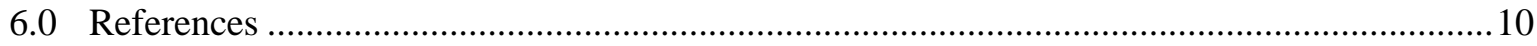

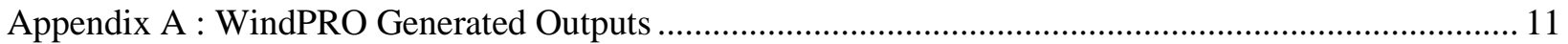


PNNL - 22926

\subsection{Introduction and Purpose}

The DeepCwind consortium, led by the University of Maine, was awarded funding under the US Department of Energy's Offshore Wind Advanced Technology Demonstration Program to develop two floating offshore wind turbines in the Gulf of Maine equipped with Goldwind 6 MW direct drive turbines, as the Aqua Ventus I project. The Goldwind turbines have a hub height of $100 \mathrm{~m}$. The turbines will be deployed in Maine State waters, approximately 2.9 miles off Monhegan Island; Monhegan Island is located roughly 10 miles off the coast of Maine.

In order to site and permit the offshore turbines, the acoustic output must be evaluated to ensure that the sound will not exceed regulatory or other established thresholds of disturbance for residents on Monhegan Island. This initial assessment of the acoustic output focuses on the sound of the turbines in air by assuming a sound source level appropriate to the proposed direct drive turbines and applying a sound propagation model to estimate sound pressure levels on Monhegan Island. 
PNNL - 22926

\subsection{Methods}

Pacific Northwest National Laboratory (PNNL) used WindPRO Decibel software to estimate the source term and propagation for sound expected to be emitted by the proposed turbines. Using several standard sound propagation models, and drawing from an international database of turbines, the WindPRO software enables the modeler to predict the outcome. Acoustic information for the Goldwind direct drive $6 \mathrm{MW}$ turbines proposed for the Aqua Ventus I project is limited and is not currently in the WindPRO database; however source level information from the turbine manufacturer (and provided to PNNL by the University of Maine) was available for the Goldwind $6 \mathrm{MW}$ direct drive turbine.

For this analysis, three large offshore wind turbines contained in the WindPRO database were used to assess the potential acoustic fields of the Goldwind $6 \mathrm{MW}$ direct drive turbines. The first two turbines used to simulate the Goldwind turbines were taken directly from the WindPRO database (a RePower 5 MW geared turbine with a hub height of $100 \mathrm{~m}$ and an ENERCON 7.5 MW direct drive turbine with a hub height of $135 \mathrm{~m}$. The third turbine was a copy of the ENERCON 7.5 MW direct drive turbine but was manually edited so that it had the same hub height $(100 \mathrm{~m})$ and source level $(115 \mathrm{~dB}(\mathrm{~A}))$ as the Goldwind $6 \mathrm{MW}$ direct drive turbine.

The WindPRO model analyzes sound propagation for a fixed wind speed of $8 \mathrm{~m} / \mathrm{s}$ using a Swedish overwater propagation model developed in 2009. This model assumes that the sound propagates spherically for the first $700 \mathrm{~m}$ and cylindrically for the remaining distance over water. It should be noted that PNNL carried out earlier acoustic modeling efforts for a different set of turbines for DeepCwind using an older Swedish water propagation model from 2002 (Naturvårdsverket 2001), which assumes spherical spreading of sound for the first $200 \mathrm{~m}$ from the source and cylindrical for the remaining distance. The older model (2002) generates higher sound pressure levels at distance greater than $200 \mathrm{~m}$ versus the 2009 model, due to the smaller radius of spherical propagation. The results from both the 2009 and 2002 Swedish sound propagation models are provided in Table 1 below for the three turbines that are the subject of this study. Water is assumed to be an acoustically hard surface with no attenuation or energy absorption by the ocean. Although this assumption is not realistic, it produces an overestimate of the received sound pressure level as it reaches Monhegan Island (i.e., is a conservative assumption). Octave band values are set at $63 \mathrm{~Hz}-4000 \mathrm{~Hz}$ and calm weather conditions are assumed. This latter assumption is important because the turbine noise is likely to be to be overpowered by wind and wave sounds in rough weather. 
PNNL - 22926

\subsection{Model Results}

The acoustic model outputs (Figures 1,2, and 3) display the sound pressure levels as a function of distance for the three representative wind turbine sources using the 2009 Swedish propagation model, relative to Monhegan Island. The source terms and estimated values for sound pressure levels for the three representative turbines using both the 2002 and 2009 Swedish sound propagation models are shown in Table 1, using the southern tip of the island as a receptor point. The WindPRO model outputs are provided in Appendix A.

Table 1. Model Inputs and Results for Three Wind Turbines used as surrogates for the Goldwind 6MW direct drive turbines proposed at Monhegan Island. Sound Pressure Level Outputs are shown for the 2002 and 2009 Swedish sound propagation models. All sound levels are recorded in an A-weighted sound pressure level in $\mathrm{dB}(\mathrm{A})$ units.

\begin{tabular}{|l|l|l|l|l|l|}
\hline Wind Turbine & Drive Train & Hub Height & Source Level & $\begin{array}{c}\text { Sound Pressure } \\
\text { Level at } \\
\text { Monhegan Island } \\
(2002 \text { model) }\end{array}$ & $\begin{array}{c}\text { Sound Pressure } \\
\text { Level at Monhegan } \\
\text { Island (2009 model) }\end{array}$ \\
\hline 5 MW REpower & Gear box & $100 \mathrm{~m}$ & $106.0 \mathrm{~dB}(\mathrm{~A})$ & $35.1 \mathrm{~dB}(\mathrm{~A})$ & $\mathbf{2 0 . 4} \mathbf{d B}(\mathbf{A})$ \\
\hline $\begin{array}{l}7.5 \mathrm{MW} \\
\text { ENERCON }\end{array}$ & Direct drive & $135 \mathrm{~m}$ & $107.5 \mathrm{~dB}(\mathrm{~A})$ & $36.5 \mathrm{~dB}(\mathrm{~A})$ & $\mathbf{2 1 . 9} \mathrm{dB}(\mathrm{A})$ \\
\hline $\begin{array}{l}7.5 \mathrm{MW} \\
\text { ENERCON V.2 } \\
\text { (edited) }\end{array}$ & Direct Drive & $100 \mathrm{~m}$ & $115 \mathrm{~dB}(\mathrm{~A})$ & $44.1 \mathrm{~dB}(\mathrm{~A})$ & $\mathbf{2 9 . 4} \mathbf{d B}(\mathbf{A})$ \\
\hline
\end{tabular}

\subsection{Discussion}

By comparing the acoustic output from the three turbines, it is likely that the $6 \mathrm{MW}$ Goldwind direct drive turbines planned for the Aqua Ventus I project will contribute approximately 20 to $29 \mathrm{~dB}(\mathrm{~A})$ to background noise at the southern tip of Monhegan Island. The sound received at the south end of Monhegan Island will decrease as it travels further over the land. It should be noted that the predicted sound pressure levels for all the modeled turbines fall below the World Health Organization's recommendation for maximum night time noise levels in Europe (40 $\mathrm{dB}(\mathrm{A})$ ) (World Health Organization 2009). To put these sound pressure levels in perspective, $30 \mathrm{~dB}$ is the background noise level of a recording studio and $40 \mathrm{~dB}$ is the level of a bedroom at night (US Department of Labor 2013); or the equivalent of a whisper.

Using the WindPRO model outputs, the use of the surrogate turbines, and taking into account the probable overestimation of acoustic propagation from the offshore sources, it is probable that the sound of the turbines reaching Monhegan Island will be very low and will almost certainly be masked at the shoreline by wave action and by ambient noise on land. 
PNNL - 22926

\subsection{Conclusion}

As the Aqua Ventus 1 project moves forward, the sound model prediction should be validated with acoustic output data from the 6MW Goldwind direct drive turbines over additional wind speeds and octaves. If such data are not available and integrated with acoustic databases such as that of WindPRO prior to deployment, additional modeling efforts for $6 \mathrm{MW}$ direct drive turbines should be undertaken to ensure that the model continues to accurately reflect the sound source levels at the turbines. 



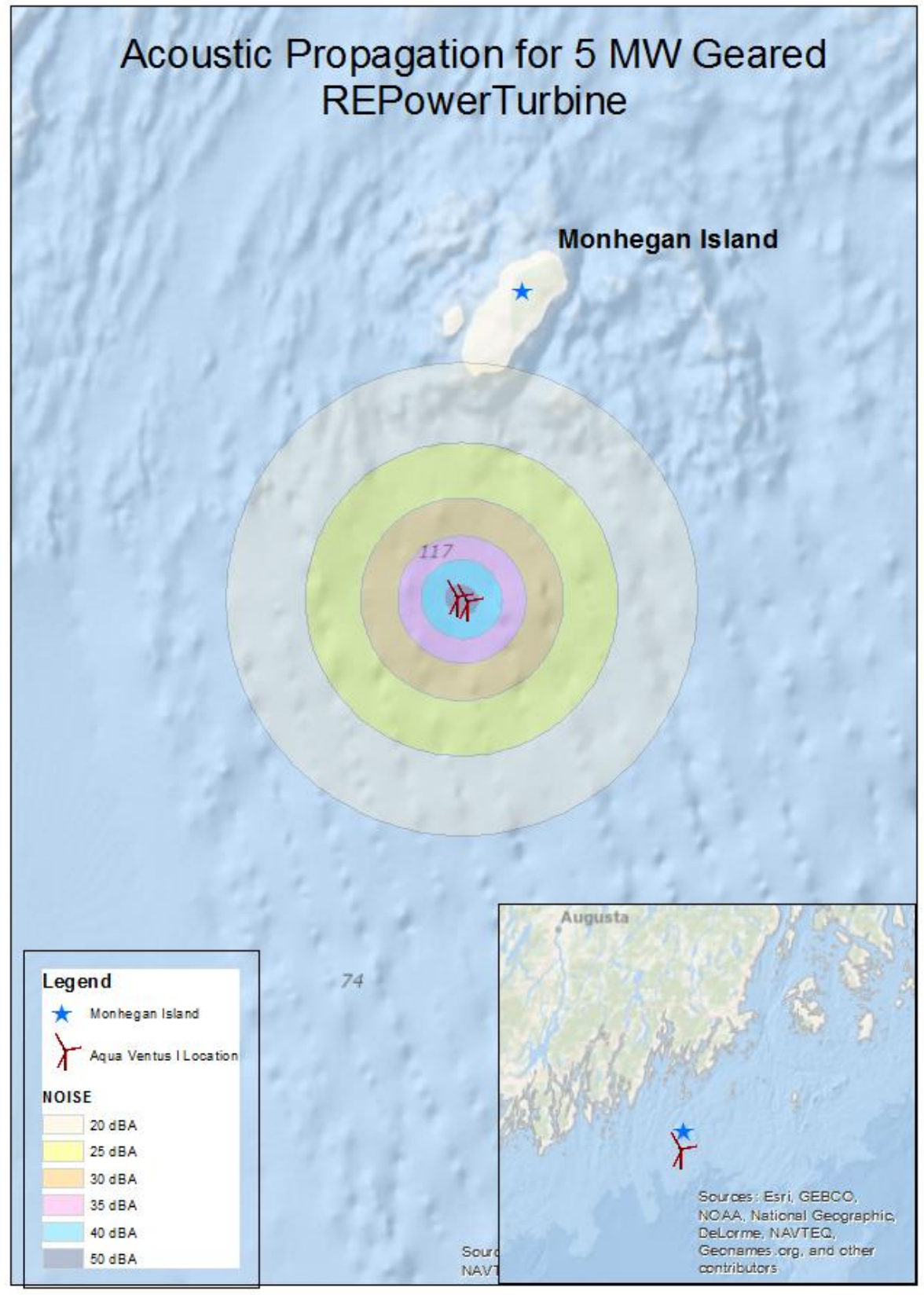

Figure 1. Model output for two 5 MW REPower geared turbines offshore of Monhegan Island ME. The wind turbines are represented by the red figures at the center of the circles. The concentric circles represent received sound levels at a distance from the source. 


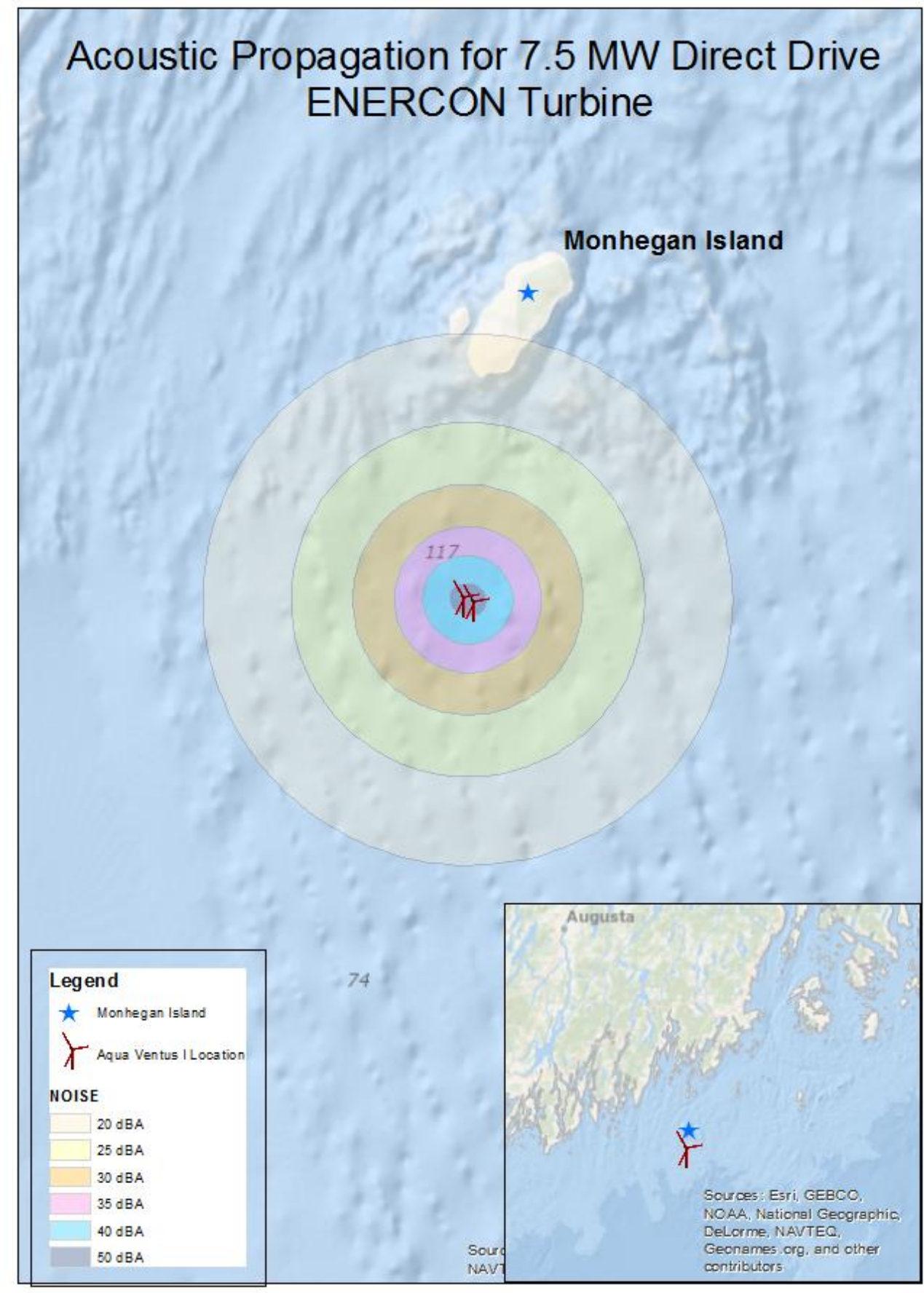

Figure 2. Model output for two 7.5 MW ENERCON direct drive turbines offshore of Monhegan Island ME. The wind turbines are represented by the red figures at the center of the circles. The concentric circles represent received sound levels at a distance from the source. 


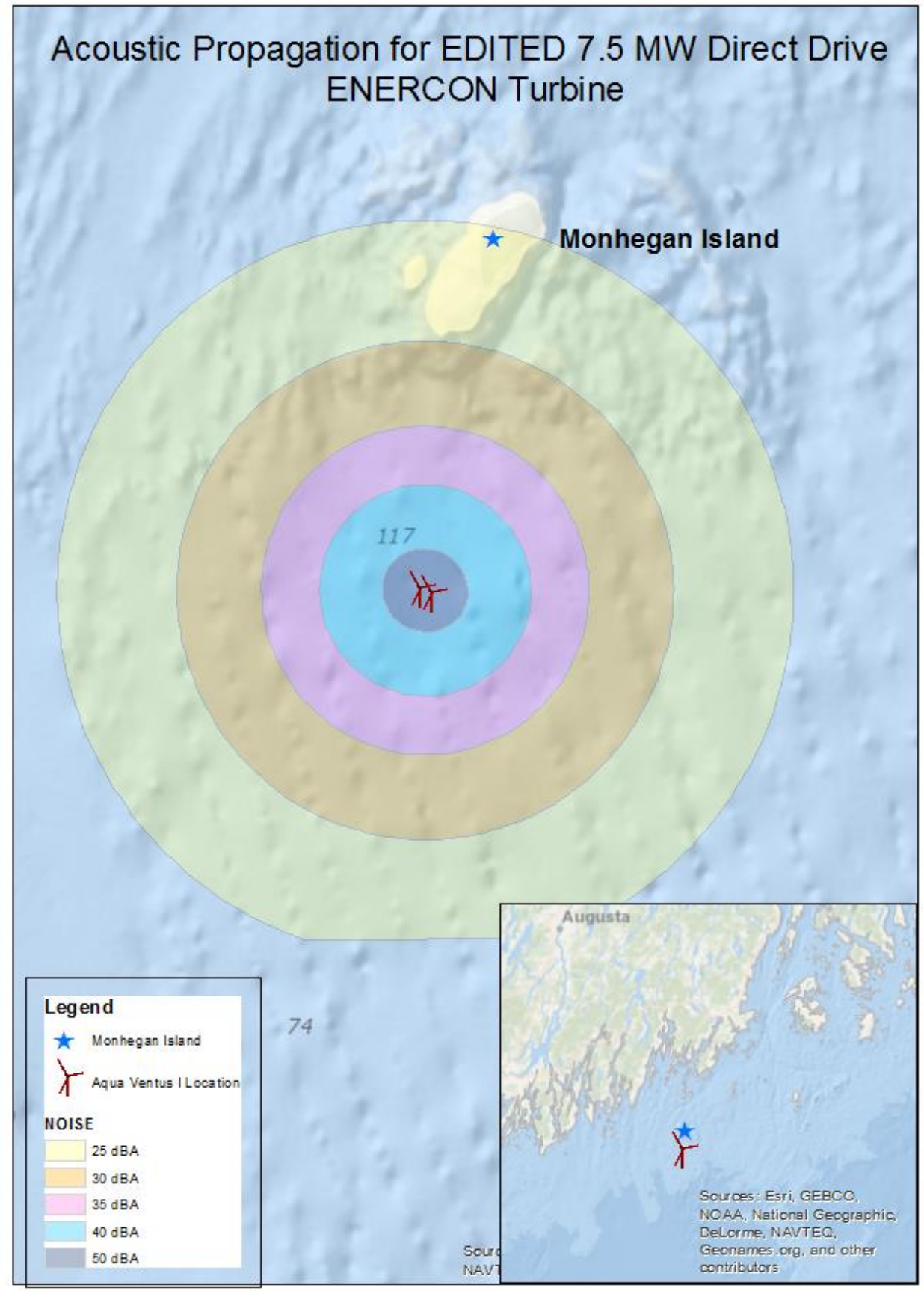

Figure 3. Model output for two adapted 7.5 MW ENERCON direct drive turbines offshore of Monhegan Island $\mathrm{ME}$ (hub height $=100 \mathrm{~m}$ and source level $=115 \mathrm{~dB}(\mathrm{~A})$ ). The wind turbines are represented by the red figures at the center of the circles. The concentric circles represent received sound levels at a distance from the source. 


\subsection{References}

Naturvårdsverket. 2001. Ljud från Vindkraftverk. Swedish EPA. ISBN 91-620-6241-7

U.S. Department of Labor: Occupational Noise Exposure. U.S. Department of Labor.

<https://www.osha.gov/SLTC/noisehearingconservation/index.html > Last accessed: 9/25/2013

World Health Organization, 2009. Night Noise Guidelines for Europe. Copenhagen, Denmark. World Health Organization. < http://www.euro.who.int/_data/assets/pdf_file/0017/43316/E92845.pdf > Last Accessed:

$10 / 21 / 2013$ 


\section{Appendix A: WindPRO Generated Outputs}

Model output for sound propagation generated by the 7.5 MW direct drive ENERCON turbine, the $5 \mathrm{MW}$ REPower geared turbine, and the edited version of the 7.5 MW direct drive ENERCON turbine offshore of Monhegan Island, ME. The wind turbines are represented by the red figures at the center of the circles. The concentric circles represent received sound levels at a distance from the source. Note: point A represents the entire Island of Monhegan; recoded sound pressure levels were taken from the closest point to the turbines on the Island. 


\begin{tabular}{|c|c|c|c|}
\hline $\begin{array}{l}\text { Project: } \\
\text { Monhegan Island Turbine Noise } 2013\end{array}$ & $\begin{array}{l}\text { Description: } \\
\text { Two } 6.0 \mathrm{MW} \text { Goldwind floating offshore wind } \\
\text { turbines are planned approximately } 3 \text { nautical } \\
\text { miles offshore from Monhegan Island, Maine. } \\
\text { Inhabitats have expressed concern with the } \\
\text { noise levels, prompting this study. } \\
\text { The } 2009 \text { Swedish code is a revision of the } \\
\text { Swedish } 2002 \text { code. A roughness coefficient } \\
\text { is replaced with a standard IEC profile of } 0.05 \\
\text { m. The distance at which geometric spreading } \\
\text { changes from spherical to cylindrical changed } \\
\text { from } 200 \mathrm{~m} \text { to } 700 \mathrm{~m} \text {. }\end{array}$ & $\begin{array}{l}\text { Printed/Page } \\
\text { 10/17/2013 10:20 AM / } 1 \\
\text { Licensed user: } \\
\text { Battelle Seattle Research Center } \\
\text { 1100 Dexter Avenue North } 98109 \\
\text { US-98109 Seattle WA } \\
\text { 509 } 3752121 \\
\text { Luke Hanna / Luke.Hanna@pnnl.gov } \\
\text { Calculated: } \\
\text { 10/17/2013 10:20 AM/2.9.250 }\end{array}$ & $\begin{array}{l}\text { Pacific Northwest } \\
\text { NATIONAL LABORATORY } \\
\text { Proudly Operated by Bamelle Since } 1965\end{array}$ \\
\hline
\end{tabular}

\section{DECIBEL - Main Result}

Calculation: RE 5 MW Standard

SWEDISH RULES FOR NOISE CALCULATION.

The calculation is based on the "Ljud frånvindkraftverk", 2010 (NV dnr 382-6897-07 Rv)

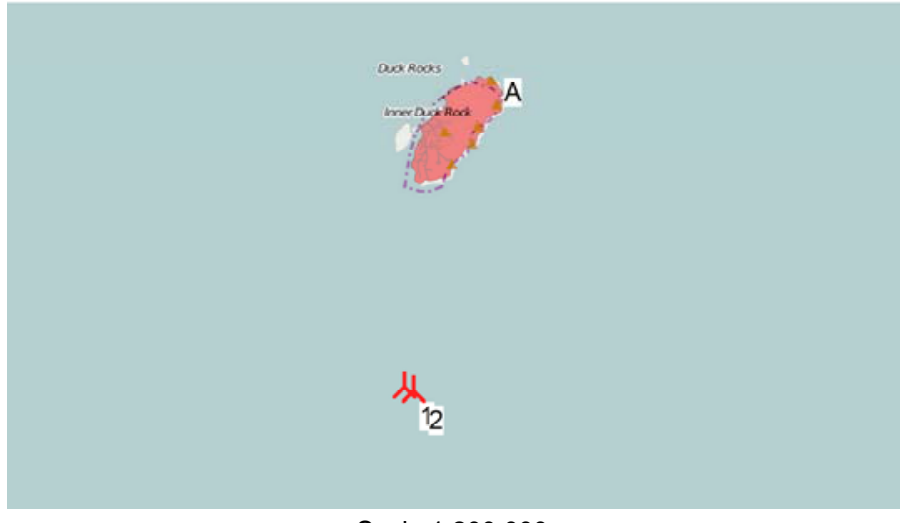

Scale 1:200,000

人 New WTG
Noise sensitive area

\section{WTGs}

Geo [deg]-WGS84

Longitude
[deg]-WG

Latitude $\quad$ Z Row data/Description

WTG type $\quad$ Noise data rated diameter height

$1-69.325371^{\circ}$ East $43.718239^{\circ}$ North 0.0 REpower 5 M 5000126.0 !O! hu... Yes REpower $5 \mathrm{M}-5,000$

126.0 1000 EMD Level 0 - guaranteed - 108,0dB(A) - 11/2008

$5,000 \quad 126.0-100.0$ EMD

2 - $69.323052^{\circ}$ East $43.717498^{\circ} \mathrm{North} 0.0 \mathrm{REpower} 5 \mathrm{M} 5000126.0$ ! O hu... Yes REpower $5 \mathrm{M}-5,000$

g) Data calculated from data for other wind speed (uncertain)

Calculation Results

\section{Sound Level}

Noise sensitive area

No. Name

Geo [deg]-WGS84
Longitude

Latitude

$Z$ Imission Noise

[m]

A Noise sensitive area: Main Island
Sound Level

From Distance to

WTGs

demand

emand

[m]
Demands fulfilled?

Noise

Yes

\section{Distances (m)}

WTG A

14161

24232 


\begin{tabular}{|c|c|c|c|}
\hline $\begin{array}{l}\text { Project: } \\
\text { Monhegan Island Turbine Noise } 2013\end{array}$ & $\begin{array}{l}\text { Description: } \\
\text { Two } 6.0 \mathrm{MW} \text { Goldwind floating offshore wind } \\
\text { turbines are planned approximately } 3 \text { nautical } \\
\text { miles offshore from Monhegan Island, Maine. } \\
\text { Inhabitats have expressed concern with the } \\
\text { noise levels, prompting this study. } \\
\text { The } 2009 \text { Swedish code is a revision of the } \\
\text { Swedish } 2002 \text { code. A roughness coefficient } \\
\text { is replaced with a standard IEC profile of } 0.05 \\
\mathrm{~m} \text {. The distance at which geometric spreading } \\
\text { changes from spherical to cylindrical changed } \\
\text { from } 200 \mathrm{~m} \text { to } 700 \mathrm{~m} \text {. }\end{array}$ & $\begin{array}{l}\text { Printed/Page } \\
\text { 10/17/2013 10:20 AM / } 2 \\
\text { Licensed user: } \\
\text { Battelle Seattle Research Center } \\
\text { 1100 Dexter Avenue North } 98109 \\
\text { US-98109 Seattle WA } \\
\text { 509 } 3752121 \\
\text { Luke Hanna / Luke.Hanna@pnnl.gov } \\
\text { Calculated: } \\
\text { 10/17/2013 10:20 AM/2.9.250 }\end{array}$ & $\begin{array}{l}\text { Pacific Northwest } \\
\text { NATIONAL LABORATORY } \\
\text { Proudly Operated by Banlelle Since } 1965\end{array}$ \\
\hline
\end{tabular}

\section{DECIBEL - Detailed results}

Calculation: RE 5 MW StandardNoise calculation model: Swedish $20098.0 \mathrm{~m} / \mathrm{s}$

Assumptions

Calculated $L(D W)=L W A, r e f+K+$ Dc $-($ Adiv + Aatm + Agr + Abar + Amisc $)-$ Cmet

(when calculated with ground attenuation, then $\mathrm{Dc}=$ Domega)

LWA,ref: $\quad$ Sound pressure level at WTG

$\mathrm{K}$ :

Pure tone

Dc:

Directivity correction

Adiv:

the attenuation due to geometrical divergence

Aatm:

Agr:

the attenuation due to atmospheric absorption

Abar:

the attenuation due to ground effect

Amisc:

the attenuation due to a barrier

Cmet:

the attenuation due to miscellaneous other effects

Meteorological correction

\section{Calculation Results}

\section{Noise sensitive area: A Noise sensitive area: Main Island}

WTG

Wind speed: $8.0 \mathrm{~m} / \mathrm{s}$

No. Distance Sound distance Land distance Calculated LwA,ref Dc Adiv Aatm Agr Abar Amisc A Cmet

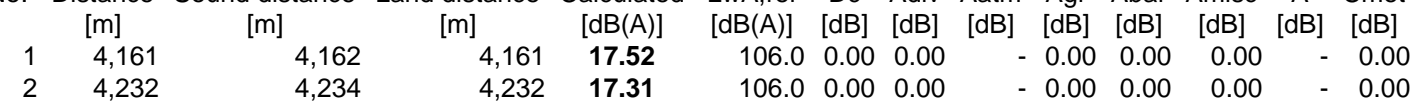

Sum 20.42

- Data undefined due to calculation with octave data 


\begin{tabular}{|c|c|c|c|}
\hline Moject: & $\begin{array}{l}\text { Description: } \\
\text { Two } 6.0 \mathrm{MW} \text { Goldwind floating offshore wind } \\
\text { turbines are planned approximately } 3 \text { nautical } \\
\text { miles offshore from Monhegan Island, Maine. } \\
\text { Inhabitats have expressed concern with the } \\
\text { noise levels, prompting this study. } \\
\text { The } 2009 \text { Swedish code is a revision of the } \\
\text { Swedish } 2002 \text { code. A roughness coefficient } \\
\text { is replaced with a standard IEC profile of } 0.05 \\
\text { m. The distance at which geometric spreading } \\
\text { changes from spherical to cylindrical changed } \\
\text { from } 200 \mathrm{~m} \text { to } 700 \mathrm{~m} \text {. }\end{array}$ & $\begin{array}{l}\text { Printed/Page } \\
\text { 10/17/2013 10:20 AM / } 3 \\
\text { Licensed user: } \\
\text { Battelle Seattle Research Center } \\
\text { 1100 Dexter Avenue North } 98109 \\
\text { US-98109 Seattle WA } \\
\text { 509 } 3752121 \\
\text { Luke Hanna / Luke.Hanna@pnnl.gov } \\
\text { Calculated: } \\
\text { 10/17/2013 10:20 AM/2.9.250 }\end{array}$ & $\begin{array}{l}\text { Pacific Northwest } \\
\text { NATIONAL LABORATORY } \\
\text { Proudly Operated by Bamene Since } 1965\end{array}$ \\
\hline
\end{tabular}

\section{DECIBEL - Assumptions for noise calculation}

\section{Calculation: RE 5 MW StandardNoise calculation model: Swedish 20098.0 m/s}

Noise calculation model:

Swedish 2009

Wind speed:

$8.0 \mathrm{~m} / \mathrm{s}$

Ground attenuation:

None

Meteorological coefficient, $\mathrm{CO}$ :

$0.0 \mathrm{~dB}$

Type of demand in calculation:

1: WTG noise is compared to demand (DK, DE, SE, NL etc.)

Noise values in calculation:

All noise values are mean values (Lwa) (Normal)

Pure tones:

Pure tone penalty are added to demand: $5.0 \mathrm{~dB}(\mathrm{~A})$

Height above ground level, when no value in NSA object:

$1.5 \mathrm{~m}$ Don't allow override of model height with height from NSA object

Deviation from "official" noise demands. Negative is more restrictive, positive is less restrictive.

$0.0 \mathrm{~dB}(\mathrm{~A})$

Octave data required

Air absorption

$\begin{array}{llllllll}63 & 125 & 250 & 500 & 1,000 & 2,000 & 4,000 & 8,000\end{array}$

$[\mathrm{db} / \mathrm{km}][\mathrm{db} / \mathrm{km}][\mathrm{db} / \mathrm{km}][\mathrm{db} / \mathrm{km}][\mathrm{db} / \mathrm{km}][\mathrm{db} / \mathrm{km}][\mathrm{db} / \mathrm{km}][\mathrm{db} / \mathrm{km}]$
0.1
0.3
0.6
1.4
$3.2 \quad 7.9$
$22.0 \quad 50.0$

WTG: REpower 5 M 5000126.0 !O!

Noise: Level 0 - guaranteed - 108,0dB(A) - 11/2008

Source Source/Date Creator Edited

REpower 11/4/2008 EMD 9/8/2009 3:17 PM

Based on documents SD-5.1_WT.SL.00-A-A-DE and SD-5.1-WT SL.01-A-B-EN.

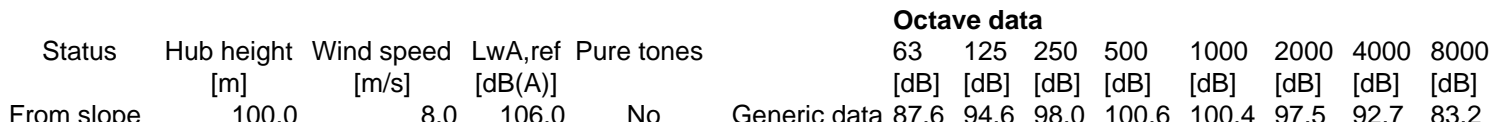

NSA: Noise sensitive area: Main Island-A

Predefined calculation standard:

Imission height(a.g.I.): $1.5 \mathrm{~m}$

Noise demand: $44.0 \mathrm{~dB}(\mathrm{~A})$

Distance demand: 


\begin{tabular}{ll}
\hline Project: & $\begin{array}{l}\text { Description: } \\
\text { Monhegan Island Turbine Noise 2013 }\end{array}$ \\
$\begin{array}{l}\text { Two } 6.0 \mathrm{MW} \text { Goldwind floating offshore wind } \\
\text { turbines are planned approximately } 3 \text { nautical } \\
\text { miles offshore from Monhegan Island, Maine. } \\
\text { Inhabitats have expressed concern with the }\end{array}$ \\
noise levels, prompting this study. \\
The 2009 Swedish code is a revision of the \\
Swedish 2002 code. A roughness coefficient \\
is replaced with a standard IEC profile of 0.05 \\
m. The distance at which geometric spreading \\
changes from spherical to cylindrical changed \\
from $200 \mathrm{~m}$ to $700 \mathrm{~m}$.
\end{tabular}

Printed/Page

10/17/2013 10:20 AM / 4

Licensed user:

Battelle Seattle Research Center

1100 Dexter Avenue North 98109

US-98109 Seattle WA

5093752121

Luke Hanna / Luke.Hanna@pnnl.gov

10/17/2013 10:20 AM/2.9.250

\section{DECIBEL - Map $8.0 \mathrm{~m} / \mathrm{s}$}

Calculation: RE 5 MW StandardNoise calculation model: Swedish $20098.0 \mathrm{~m} / \mathrm{s}$

Noise $[\mathrm{dB}(\mathrm{A})]$

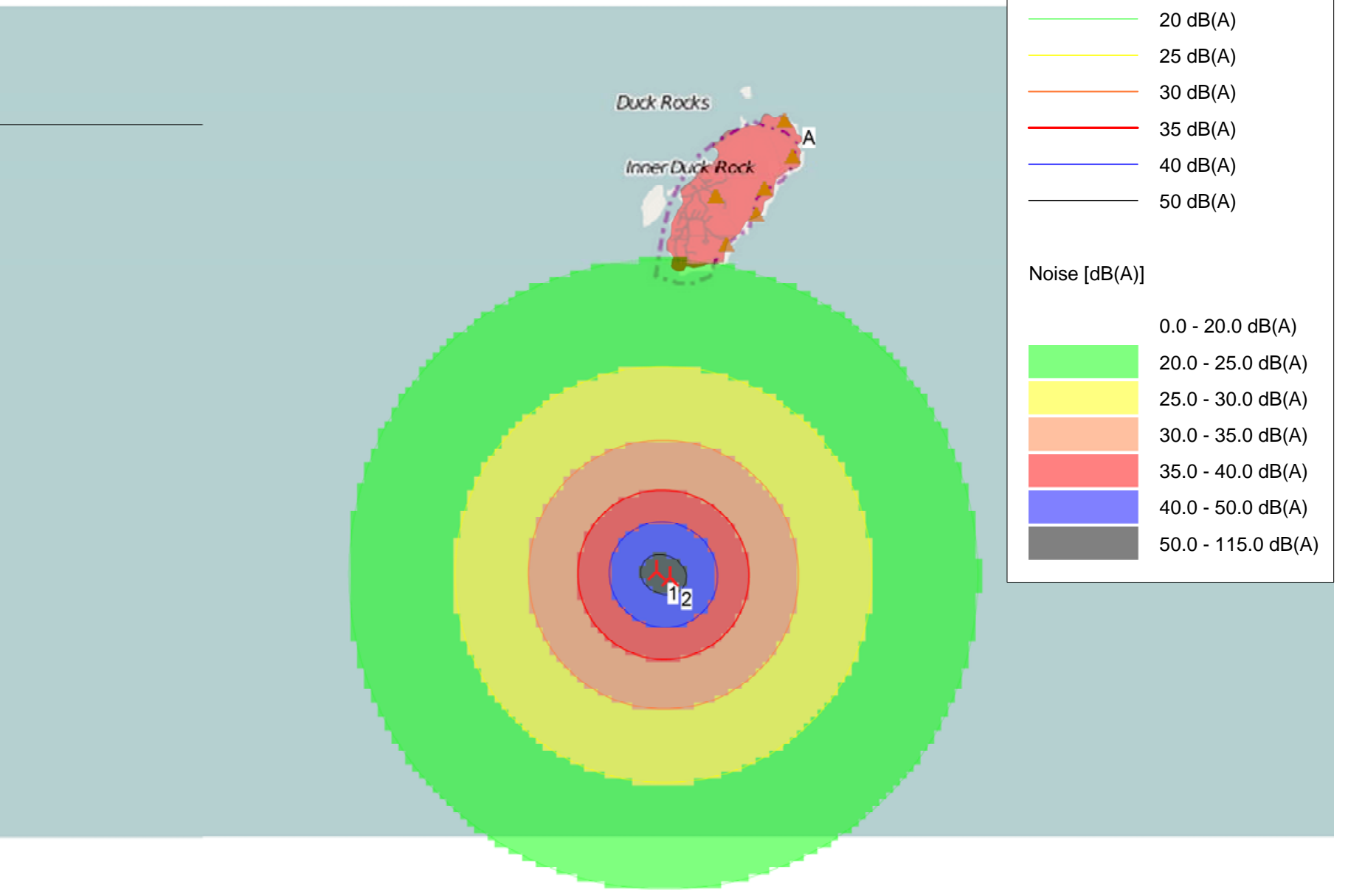

人 New WTG

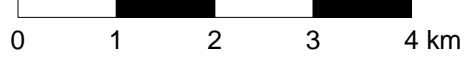

Map: WindPRO map , Print scale 1:100,000, Map center Geo WGS84 East: $-69.324144^{\circ}$ East North: $43.719168^{\circ}$ North

Noise sensitive area

Noise calculation model: Swedish 2009. Wind speed: $8.0 \mathrm{~m} / \mathrm{s}$

Height above sea level from active line object 


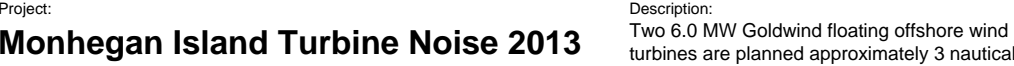 miles offshore from Monhegan Island, Maine. Inhabitats have expressed concern with the noise levels, prompting this study. \\ The 2009 Swedish code is a revision of the \\ Swedish 2002 code. A roughness coefficient \\ is replaced with a standard IEC profile of 0.05 \\ $\mathrm{m}$. The distance at which geometric spreading \\ changes from spherical to cylindrical changed \\ from $200 \mathrm{~m}$ to $700 \mathrm{~m}$.}

Printed/Page

10/17/2013 10:18 AM / 1

Licensed user:

Battelle Seattle Research Center

1100 Dexter Avenue North 98109

US-98109 Seattle WA

5093752121

Luke Hanna / Luke.Hanna@pnnl.gov

10/17/2013 10:17 AM/2.9.250

\section{DECIBEL - Main Result}

\section{Calculation: ENERCON 7.5 MW Standard}

SWEDISH RULES FOR NOISE CALCULATION.

The calculation is based on the "Ljud frånvindkraftverk", 2010 (NV dnr 382-6897-07 Rv)

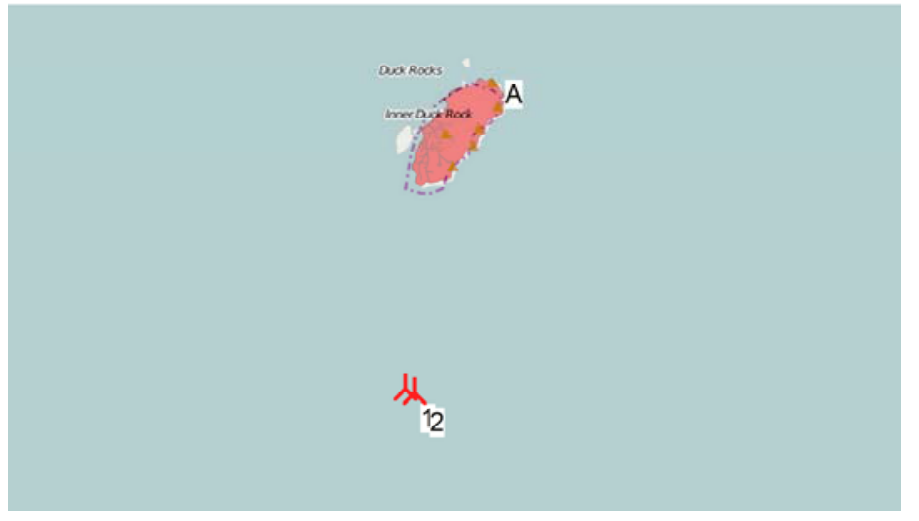

Scale 1:200,000

人 New WTG Noise sensitive area

\section{WTGs}

Geo [deg]-WGS84

Longitude

Latitude $\quad$ Z Row data/Description

[m]

$1-69.325371^{\circ}$ East $43.718239^{\circ}$ North 0.0 ENERCON E-126 $7500127.0 \ldots$ Yes ENERCON E-126-7,500

$2-69.323052^{\circ}$ East $43.717498^{\circ}$ North 0.0 ENERCON E-126 $7500127.0 \ldots$ Yes ENERCON E-126-7,500

h) Generic octave distribution used

Calculation Results

Sound Level

Noise sensitive area

No. Name

A Noise sensitive area: Main Island
WTG type

Valid Manufact. Type-generator Power, Rotor

Power, Rotor Hub
rated diameter height

$\begin{array}{ll}\text { rated diam } \\ {[\mathrm{kW}]} & {[\mathrm{m}]} \\ 7,500 & 127.0\end{array}$

$7,500 \quad 127.0$

$7,500 \quad 127.0$
Noise data

Creator Name

m

[m]

135.0 EMD
Level 0 - calculated - Op.Mode I - 12/2010 Level 0 - calculated - Op.Mode I - 12/2010
Pacific Northwest

NATONAL LABORATORY

Proudly Operated by Banele Simce 196

\section{Distances (m)}

WTG A

14161

24232
Geo [deg]-WGS84

Longitude

Latitude

\section{Z Imission height}

[m]

$69.321328^{\circ}$ East $43.755586^{\circ}$ North 39.5 [m] ${ }_{1.5}$
Demands Sound Level

Noise From Distance to

WTGs noise

demand

$[\mathrm{dB}(\mathrm{A})] \quad[\mathrm{dB}(\mathrm{A})] \quad[\mathrm{m}]$
Demands fulfilled?

Noise

Yes
Wind LwA,ref Pure

$[\mathrm{m} / \mathrm{s}] \quad[\mathrm{dB}(\mathrm{A})]$

107.5 No $h$ 8.0107 .5 No $h$ 


\begin{tabular}{|c|c|c|c|}
\hline $\begin{array}{l}\text { Project: } \\
\text { Monhegan Island Turbine Noise } 2013\end{array}$ & $\begin{array}{l}\text { Description: } \\
\text { Two } 6.0 \mathrm{MW} \text { Goldwind floating offshore wind } \\
\text { turbines are planned approximately } 3 \text { nautical } \\
\text { miles offshore from Monhegan Island, Maine. } \\
\text { Inhabitats have expressed concern with the } \\
\text { noise levels, prompting this study. } \\
\text { The } 2009 \text { Swedish code is a revision of the } \\
\text { Swedish } 2002 \text { code. A roughness coefficient } \\
\text { is replaced with a standard IEC profile of } 0.05 \\
\text { m. The distance at which geometric spreading } \\
\text { changes from spherical to cylindrical changed } \\
\text { from } 200 \mathrm{~m} \text { to } 700 \mathrm{~m} \text {. }\end{array}$ & $\begin{array}{l}\text { Printed/Page } \\
\text { 10/17/2013 10:18 AM / } 2 \\
\text { Licensed user: } \\
\text { Battelle Seattle Research Center } \\
\text { 1100 Dexter Avenue North } 98109 \\
\text { US-98109 Seattle WA } \\
5093752121 \\
\text { Luke Hanna / Luke.Hanna@pnnl.gov } \\
\text { Calculated: } \\
\text { 10/17/2013 10:17 AM/2.9.250 }\end{array}$ & $\begin{array}{l}\text { Pacific Northwest } \\
\text { NATONAL LABORATORY } \\
\text { Proudly Operated by Banlelle Simce } 1965\end{array}$ \\
\hline
\end{tabular}

\section{DECIBEL - Detailed results}

Calculation: ENERCON 7.5 MW StandardNoise calculation model: Swedish $20098.0 \mathrm{~m} / \mathrm{s}$

Assumptions

Calculated $L(D W)=L W A, r e f+K+$ Dc $-($ Adiv + Aatm + Agr + Abar + Amisc $)-$ Cmet

(when calculated with ground attenuation, then $\mathrm{Dc}=$ Domega)

LWA,ref: $\quad$ Sound pressure level at WTG

$\mathrm{K}$ :

Pure tone

Dc:

Directivity correction

Adiv:

the attenuation due to geometrical divergence

Aatm:

Agr:

the attenuation due to atmospheric absorption

Abar:

the attenuation due to ground effect

Amisc:

the attenuation due to a barrier

Cmet:

the attenuation due to miscellaneous other effects

Meteorological correction

\section{Calculation Results}

\section{Noise sensitive area: A Noise sensitive area: Main Island}

WTG

Wind speed: $8.0 \mathrm{~m} / \mathrm{s}$

No. Distance Sound distance Land distance Calculated LwA,ref Dc Adiv Aatm Agr Abar Amisc A Cmet

\begin{tabular}{|c|c|c|c|c|c|c|c|c|c|c|c|}
\hline [m] & {$[\mathrm{m}]$} & {$[\mathrm{m}]$} & {$[\mathrm{dB}(\mathrm{A})]$} & {$[\mathrm{dB}(\mathrm{A})]$} & {$[\mathrm{dB}]$} & [dB] & [dB] & [dB] & {$[\mathrm{dB}]$} & [dB] & {$[\mathrm{dB}]$} \\
\hline 4,161 & 4,163 & 4,161 & 19.01 & 107.5 & 0.00 & 0.00 & & 0.00 & 0.00 & 0.00 & 0.00 \\
\hline 4,232 & 4,235 & 4,232 & 18.80 & 107.5 & 0.00 & 0.00 & & 0.00 & 0.00 & 0.00 & 0.00 \\
\hline
\end{tabular}

Sum 21.92

- Data undefined due to calculation with octave data 


\begin{tabular}{|c|c|c|c|}
\hline Moject: & $\begin{array}{l}\text { Description: } \\
\text { Two } 6.0 \mathrm{MW} \text { Goldwind floating offshore wind } \\
\text { turbines are planned approximately } 3 \text { nautical } \\
\text { miles offshore from Monhegan Island, Maine. } \\
\text { Inhabitats have expressed concern with the } \\
\text { noise levels, prompting this study. } \\
\text { The } 2009 \text { Swedish code is a revision of the } \\
\text { Swedish } 2002 \text { code. A roughness coefficient } \\
\text { is replaced with a standard IEC profile of } 0.05 \\
\text { m. The distance at which geometric spreading } \\
\text { changes from spherical to cylindrical changed } \\
\text { from } 200 \mathrm{~m} \text { to } 700 \mathrm{~m} \text {. }\end{array}$ & $\begin{array}{l}\text { Printed/Page } \\
\text { 10/17/2013 10:18 AM / } 3 \\
\text { Licensed user: } \\
\text { Battelle Seattle Research Center } \\
\text { 1100 Dexter Avenue North } 98109 \\
\text { US-98109 Seattle WA } \\
\text { 509 } 3752121 \\
\text { Luke Hanna / Luke.Hanna@pnnl.gov } \\
\text { Calculated: } \\
\text { 10/17/2013 10:17 AM/2.9.250 }\end{array}$ & $\begin{array}{l}\text { Pacific Northwest } \\
\text { NATIONAL LABORATORY } \\
\text { Proudly Operated by Bamene Since } 1965\end{array}$ \\
\hline
\end{tabular}

\section{DECIBEL - Assumptions for noise calculation}

Calculation: ENERCON 7.5 MW StandardNoise calculation model: Swedish $20098.0 \mathrm{~m} / \mathrm{s}$

Noise calculation model:

Swedish 2009

Wind speed:

$8.0 \mathrm{~m} / \mathrm{s}$

Ground attenuation:

None

Meteorological coefficient, $\mathrm{C} 0$ :

$0.0 \mathrm{~dB}$

Type of demand in calculation:

1: WTG noise is compared to demand (DK, DE, SE, NL etc.)

Noise values in calculation:

All noise values are mean values (Lwa) (Normal)

Pure tones:

Pure tone penalty are added to demand: $5.0 \mathrm{~dB}(\mathrm{~A})$

Height above ground level, when no value in NSA object:

$1.5 \mathrm{~m}$ Don't allow override of model height with height from NSA object

Deviation from "official" noise demands. Negative is more restrictive, positive is less restrictive.:

$0.0 \mathrm{~dB}(\mathrm{~A})$

Octave data required

Air absorption

$\begin{array}{llllllll}63 & 125 & 250 & 500 & 1,000 & 2,000 & 4,000 & 8,000\end{array}$

$[\mathrm{db} / \mathrm{km}][\mathrm{db} / \mathrm{km}][\mathrm{db} / \mathrm{km}][\mathrm{db} / \mathrm{km}][\mathrm{db} / \mathrm{km}][\mathrm{db} / \mathrm{km}][\mathrm{db} / \mathrm{km}][\mathrm{db} / \mathrm{km}]$
0.1
0.3
0.6
$1.4 \quad 3.2$
7.922 .0
50.0

WTG: ENERCON E-126 7500127.0 !O!

Noise: Level 0 - calculated - Op.Mode I - 12/2010

Source Source/Date Creator Edited

Manufacturer 12/8/2010 EMD 1/7/2013 9:34 AM

According to manufacturer specification document "SIAS-04-SPL E-126 75 Est Rev1_ 0-ger-ger.pdf" dated 12/2010

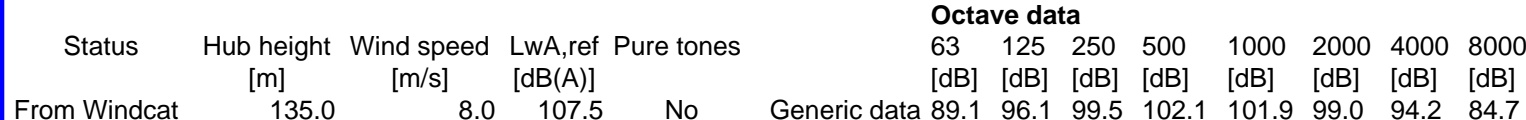

NSA: Noise sensitive area: Main Island-A

Predefined calculation standard:

Imission height(a.g.I.): $1.5 \mathrm{~m}$

Noise demand: $44.0 \mathrm{~dB}(\mathrm{~A})$

Distance demand: 


\begin{tabular}{ll}
\hline Project: & $\begin{array}{l}\text { Description: } \\
\text { Monhegan Island Turbine Noise 2013 }\end{array}$ \\
$\begin{array}{l}\text { Two } 6.0 \mathrm{MW} \text { Goldwind floating offshore wind } \\
\text { turbines are planned approximately } 3 \text { nautical } \\
\text { miles offshore from Monhegan Island, Maine. } \\
\text { Inhabitats have expressed concern with the }\end{array}$ \\
noise levels, prompting this study. \\
The 2009 Swedish code is a revision of the \\
Swedish 2002 code. A roughness coefficient \\
is replaced with a standard IEC profile of 0.05 \\
m. The distance at which geometric spreading \\
changes from spherical to cylindrical changed \\
from $200 \mathrm{~m}$ to $700 \mathrm{~m}$.
\end{tabular}

Printed/Page

10/17/2013 10:18 AM / 4

Licensed user:

Battelle Seattle Research Center

1100 Dexter Avenue North 98109

US-98109 Seattle WA

5093752121

Luke Hanna / Luke.Hanna@pnnl.gov

10/17/2013 10:17 AM/2.9.250

\section{DECIBEL - Map $8.0 \mathrm{~m} / \mathrm{s}$}

Calculation: ENERCON 7.5 MW StandardNoise calculation model: Swedish $20098.0 \mathrm{~m} / \mathrm{s}$

Noise $[\mathrm{dB}(\mathrm{A})]$

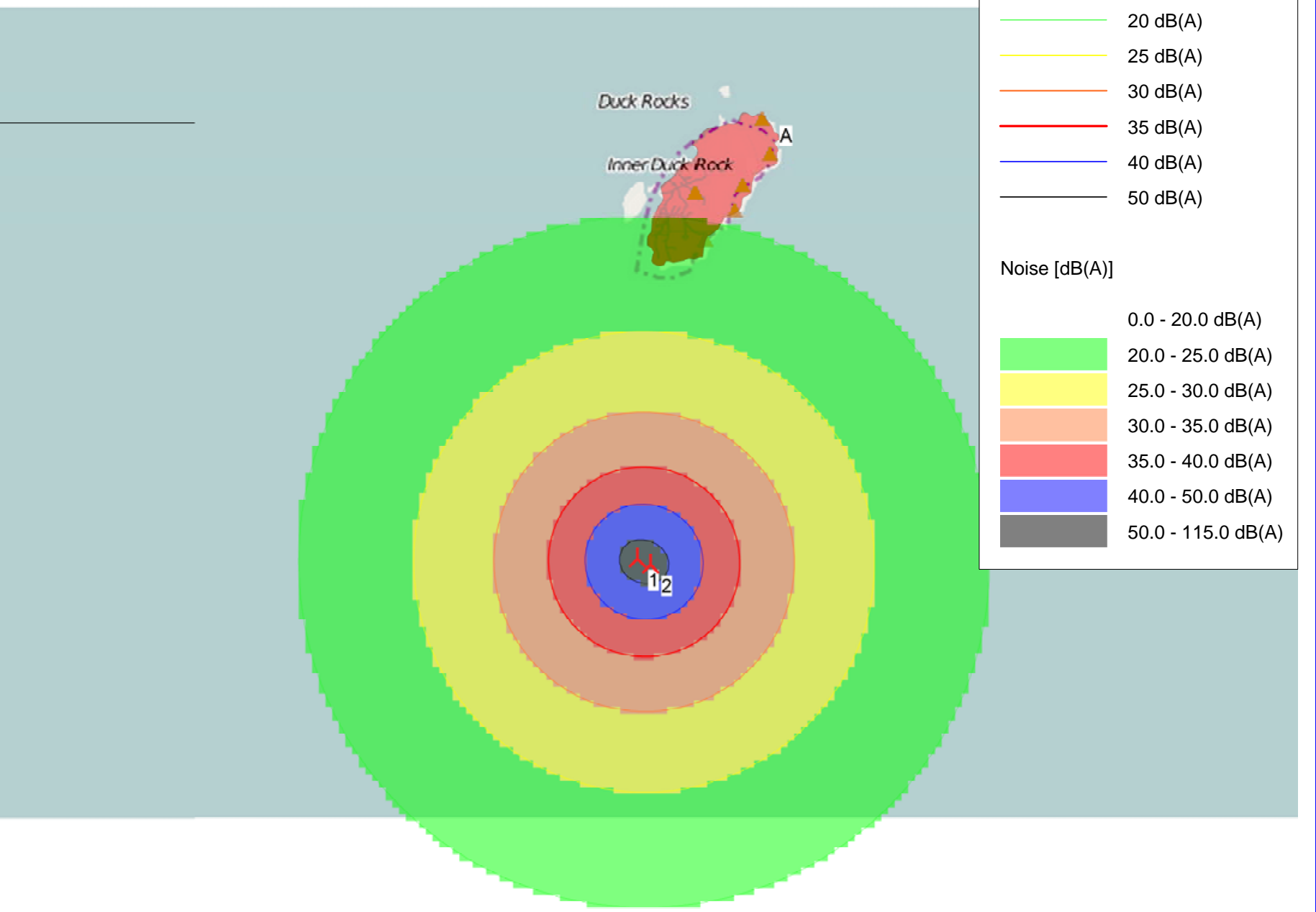

人 New WTG

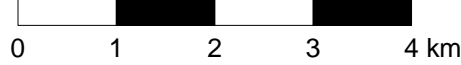

Map: WindPRO map, Print scale 1:100,000, Map center Geo WGS84 East: $-69.324144^{\circ}$ East North: $43.719168^{\circ}$ North

- Noise sensitive area

Noise calculation model: Swedish 2009. Wind speed: $8.0 \mathrm{~m} / \mathrm{s}$

Height above sea level from active line object 


\begin{tabular}{|c|c|c|c|}
\hline $\begin{array}{l}\text { Project: } \\
\text { Monhegan Island Turbine Noise } 2013\end{array}$ & $\begin{array}{l}\text { Description: } \\
\text { Two } 6.0 \mathrm{MW} \text { Goldwind floating offshore wind } \\
\text { turbines are planned approximately } 3 \text { nautical } \\
\text { miles offshore from Monhegan Island, Maine. } \\
\text { Inhabitats have expressed concern with the } \\
\text { noise levels, prompting this study. } \\
\text { The } 2009 \text { Swedish code is a revision of the } \\
\text { Swedish } 2002 \text { code. A roughness coefficient } \\
\text { is replaced with a standard IEC profile of } 0.05 \\
\text { m. The distance at which geometric spreading } \\
\text { changes from spherical to cylindrical changed } \\
\text { from } 200 \mathrm{~m} \text { to } 700 \mathrm{~m} \text {. }\end{array}$ & $\begin{array}{l}\text { Printed/Page } \\
\text { 10/17/2013 10:31 AM / } 1 \\
\text { Licensed user: } \\
\text { Battelle Seattle Research Center } \\
\text { 1100 Dexter Avenue North } 98109 \\
\text { US-98109 Seattle WA } \\
5093752121 \\
\text { Luke Hanna / Luke.Hanna@pnnl.gov } \\
\text { Calculated: } \\
\text { 10/17/2013 10:31 AM/2.9.250 }\end{array}$ & $\begin{array}{l}\text { Pacific Northwest } \\
\text { NATIONAL LABORATORY } \\
\text { Proudly Operated by Bamelle Since } 1965\end{array}$ \\
\hline
\end{tabular}

\section{DECIBEL - Main Result}

Calculation: Goldwind 6 MW Custom

SWEDISH RULES FOR NOISE CALCULATION.

The calculation is based on the "Ljud frånvindkraftverk", 2010 (NV dnr 382-6897-07 Rv)

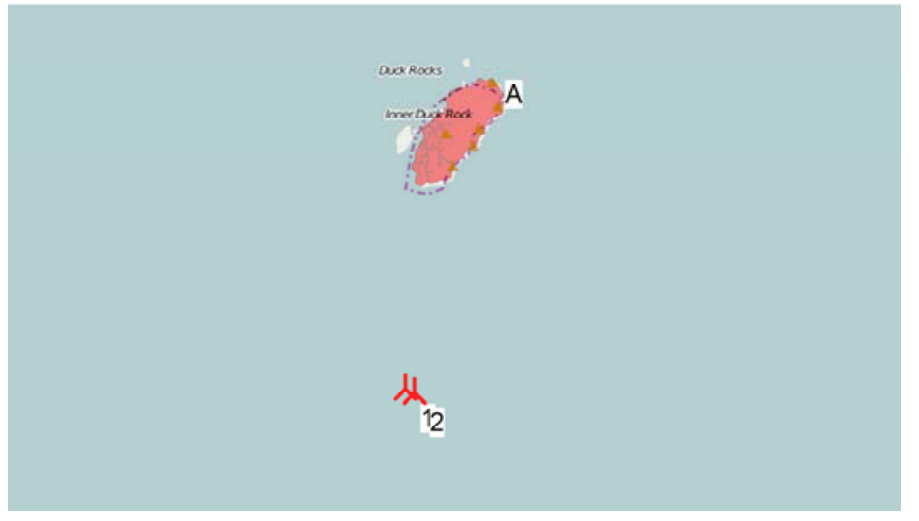

Scale 1:200,000

人 New WTG
Noise sensitive area

\section{WTGs}

Geo [deg]-WGS84

Longitude
WTG type

Valid Manufact. Type-generator \begin{tabular}{lll} 
Power, Rotor Hub & \multicolumn{2}{l}{ Noise data } \\
rated diameter height Name
\end{tabular} [kW] [m] $[\mathrm{m}]$ $[\mathrm{m}]$

$1-69.325371^{\circ}$ East $43.718239^{\circ}$ North 0.0 Goldwind GW6.0-152 $60001 \ldots$ Yes Goldwind GW6.0-152-6,000 6,000 152.

$2-69.323052^{\circ}$ East $43.717498^{\circ}$ North 0.0 Goldwind GW6.0-152 $60001 \ldots$ Yes Goldwind GW6.0-152-6,000 6,000 152.2 h) Generic octave distribution used

\section{Calculation Results}

\section{Sound Level}

Noise sensitive area

No. Name

A Noise sensitive area: Main Island

$-69.321328^{\circ}$ East
Geo [deg]-WGS84

Longitude

Latitude
Z Imission
height

[m] [m] 100.0 US

Level 0 - Estimated - $115 \mathrm{~dB}$ - 09-2013

100.0 USER Level 0 - Estimated $-115 \mathrm{~dB}-09-2013$

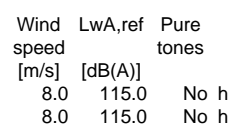

Wind LwA,ref Pure

$\mathrm{m} / \mathrm{s}] \quad[\mathrm{dB}(\mathrm{A})]$

8.0115 .0 No $h$

\section{Distances ( $m$ )}

WTG A

14161

24232 


\begin{tabular}{|c|c|c|c|}
\hline $\begin{array}{l}\text { Project: } \\
\text { Monhegan Island Turbine Noise } 2013\end{array}$ & $\begin{array}{l}\text { Description: } \\
\text { Two } 6.0 \mathrm{MW} \text { Goldwind floating offshore wind } \\
\text { turbines are planned approximately } 3 \text { nautical } \\
\text { miles offshore from Monhegan Island, Maine. } \\
\text { Inhabitats have expressed concern with the } \\
\text { noise levels, prompting this study. } \\
\text { The } 2009 \text { Swedish code is a revision of the } \\
\text { Swedish } 2002 \text { code. A roughness coefficient } \\
\text { is replaced with a standard IEC profile of } 0.05 \\
\mathrm{~m} \text {. The distance at which geometric spreading } \\
\text { changes from spherical to cylindrical changed } \\
\text { from } 200 \mathrm{~m} \text { to } 700 \mathrm{~m} \text {. }\end{array}$ & $\begin{array}{l}\text { Printed/Page } \\
\text { 10/17/2013 10:31 AM / } 2 \\
\text { Licensed user: } \\
\text { Battelle Seattle Research Center } \\
\text { 1100 Dexter Avenue North } 98109 \\
\text { US-98109 Seattle WA } \\
\text { 509 } 3752121 \\
\text { Luke Hanna / Luke.Hanna@pnnl.gov } \\
\text { Calculated: } \\
\text { 10/17/2013 10:31 AM/2.9.250 }\end{array}$ & $\begin{array}{l}\text { Pacific Northwest } \\
\text { NATIONAL LABORATORY } \\
\text { Proudly Operated by Banlelle Since } 1965\end{array}$ \\
\hline
\end{tabular}

\section{DECIBEL - Detailed results}

Calculation: Goldwind 6 MW CustomNoise calculation model: Swedish 20098.0 m/s

Assumptions

Calculated $L(D W)=L W A$, ref $+K+$ Dc $-($ Adiv + Aatm + Agr + Abar + Amisc $)-$ Cmet

(when calculated with ground attenuation, then $\mathrm{Dc}=$ Domega)

LWA,ref: $\quad$ Sound pressure level at WTG

$\mathrm{K}$ :

Pure tone

Dc:

Directivity correction

Adiv:

the attenuation due to geometrical divergence

Aatm:

the attenuation due to atmospheric absorption

Agr:

Abar:

the attenuation due to ground effect

Amisc:

the attenuation due to a barrier

Cmet:

the attenuation due to miscellaneous other effects

Meteorological correction

\section{Calculation Results}

\section{Noise sensitive area: A Noise sensitive area: Main Island}

WTG

Wind speed: $8.0 \mathrm{~m} / \mathrm{s}$

No. Distance Sound distance Land distance Calculated LwA,ref Dc Adiv Aatm Agr Abar Amisc A Cmet

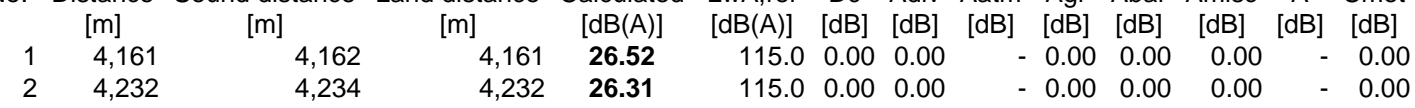

Sum 29.42

- Data undefined due to calculation with octave data 


\begin{tabular}{|c|c|c|c|}
\hline Moject: & $\begin{array}{l}\text { Description: } \\
\text { Two } 6.0 \mathrm{MW} \text { Goldwind floating offshore wind } \\
\text { turbines are planned approximately } 3 \text { nautical } \\
\text { miles offshore from Monhegan Island, Maine. } \\
\text { Inhabitats have expressed concern with the } \\
\text { noise levels, prompting this study. } \\
\text { The } 2009 \text { Swedish code is a revision of the } \\
\text { Swedish } 2002 \text { code. A roughness coefficient } \\
\text { is replaced with a standard IEC profile of } 0.05 \\
\text { m. The distance at which geometric spreading } \\
\text { changes from spherical to cylindrical changed } \\
\text { from } 200 \mathrm{~m} \text { to } 700 \mathrm{~m} \text {. }\end{array}$ & $\begin{array}{l}\text { Printed/Page } \\
\text { 10/17/2013 10:31 AM / } 3 \\
\text { Licensed user: } \\
\text { Battelle Seattle Research Center } \\
\text { 1100 Dexter Avenue North } 98109 \\
\text { US-98109 Seattle WA } \\
\text { 509 } 3752121 \\
\text { Luke Hanna / Luke.Hanna@pnnl.gov } \\
\text { Calculated: } \\
\text { 10/17/2013 10:31 AM/2.9.250 }\end{array}$ & $\begin{array}{l}\text { Pacific Northwest } \\
\text { NATIONAL LABORATORY } \\
\text { Proudly Operated by Bamene Since } 1965\end{array}$ \\
\hline
\end{tabular}

\section{DECIBEL - Assumptions for noise calculation}

Calculation: Goldwind 6 MW CustomNoise calculation model: Swedish 20098.0 m/s

Noise calculation model:

Swedish 2009

Wind speed:

$8.0 \mathrm{~m} / \mathrm{s}$

Ground attenuation:

None

Meteorological coefficient, $\mathrm{CO}$ :

$0.0 \mathrm{~dB}$

Type of demand in calculation:

1: WTG noise is compared to demand (DK, DE, SE, NL etc.)

Noise values in calculation:

All noise values are mean values (Lwa) (Normal)

Pure tones:

Pure tone penalty are added to demand: $5.0 \mathrm{~dB}(\mathrm{~A})$

Height above ground level, when no value in NSA object:

$1.5 \mathrm{~m}$ Don't allow override of model height with height from NSA object

Deviation from "official" noise demands. Negative is more restrictive, positive is less restrictive.:

$0.0 \mathrm{~dB}(\mathrm{~A})$

Octave data required

Air absorption

$\begin{array}{llllllll}63 & 125 & 250 & 500 & 1,000 & 2,000 & 4,000 & 8,000\end{array}$

$[\mathrm{db} / \mathrm{km}][\mathrm{db} / \mathrm{km}][\mathrm{db} / \mathrm{km}][\mathrm{db} / \mathrm{km}][\mathrm{db} / \mathrm{km}][\mathrm{db} / \mathrm{km}][\mathrm{db} / \mathrm{km}][\mathrm{db} / \mathrm{km}]$
0.1
0.3
0.6
$1.4 \quad 3.2$
7.922 .0
50.0

WTG: Goldwind GW6.0-152 6000152.2 !O!

Noise: Level 0 - Estimated - $115 \mathrm{~dB}$ - 09-2013

Source Source/Date Creator Edited

Manufacturer 9/20/2013 USER 10/3/2013 1:08 PM

Based on Goldwind 128/6000 Wind Turbine Specification

Status Hub height Wind speed LwA,ref Pure tones

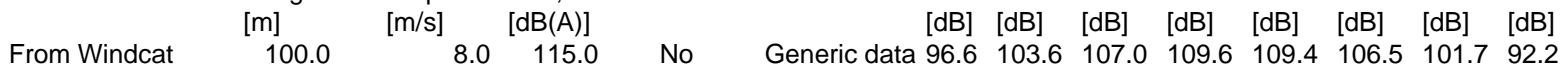

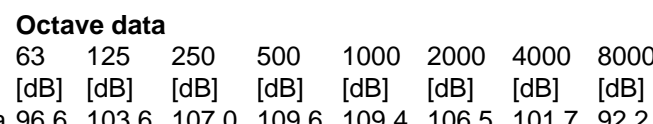

Octave data

From Windcat

100.0

$8.0 \quad 115.0$

No

NSA: Noise sensitive area: Main Island-A

Predefined calculation standard:

Imission height(a.g.I.): $1.5 \mathrm{~m}$

Noise demand: $44.0 \mathrm{~dB}(\mathrm{~A})$

Distance demand: 


\begin{tabular}{|c|c|}
\hline $\begin{array}{l}\text { Project: } \\
\text { Monhegan Island Turbine Noise } 2013\end{array}$ & $\begin{array}{l}\text { Description: } \\
\text { Two } 6.0 \mathrm{MW} \text { Goldwind floating offshore wind } \\
\text { turbines are planned approximately } 3 \text { nautical } \\
\text { miles offshore from Monhegan Island, Maine. } \\
\text { Inhabitats have expressed concern with the } \\
\text { noise levels, prompting this study. } \\
\text { The } 2009 \text { Swedish code is a revision of the } \\
\text { Swedish } 2002 \text { code. A roughness coefficient } \\
\text { is replaced with a standard IEC profile of } 0.05 \\
\text { m. The distance at which geometric spreading } \\
\text { changes from spherical to cylindrical changed } \\
\text { from } 200 \mathrm{~m} \text { to } 700 \mathrm{~m} \text {. }\end{array}$ \\
\hline
\end{tabular}

Printed/Page

10/17/2013 10:31 AM / 4

Licensed user:

Battelle Seattle Research Center

1100 Dexter Avenue North 98109

US-98109 Seattle WA

5093752121

Luke Hanna / Luke.Hanna@pnnl.gov

10/17/2013 10:31 AM/2.9.250

\section{DECIBEL - Map $8.0 \mathrm{~m} / \mathrm{s}$}

Calculation: Goldwind 6 MW CustomNoise calculation model: Swedish $20098.0 \mathrm{~m} / \mathrm{s}$

Noise $[\mathrm{dB}(\mathrm{A})]$

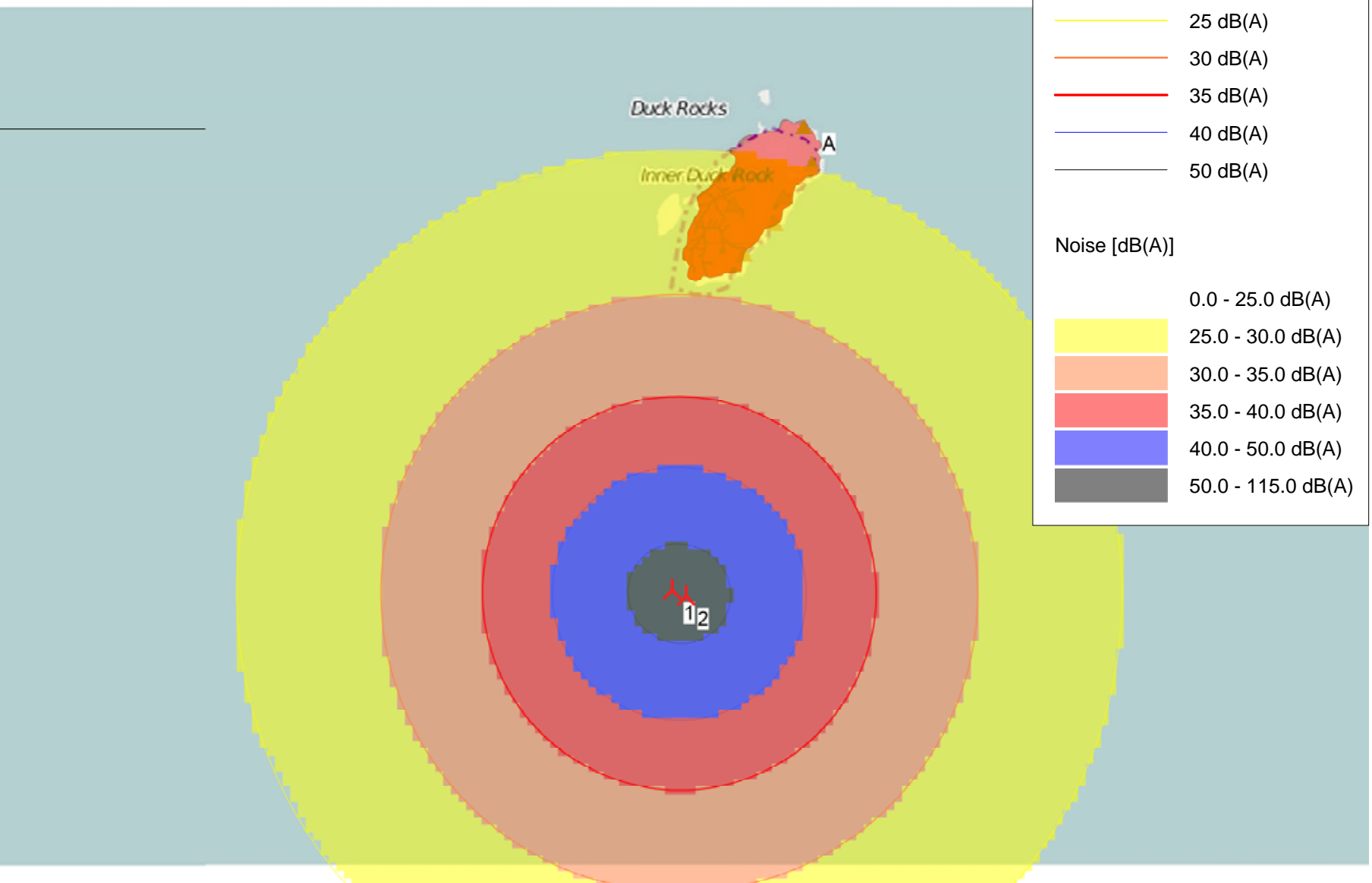

New WTG Noise sensitive area

Map: WindPRO map , Print scale 1:100,000, Map center Geo WGS84 East: $-69.324132^{\circ}$ East North: $43.717841^{\circ}$ North

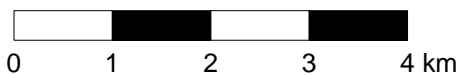

Height above sea level from active line object 


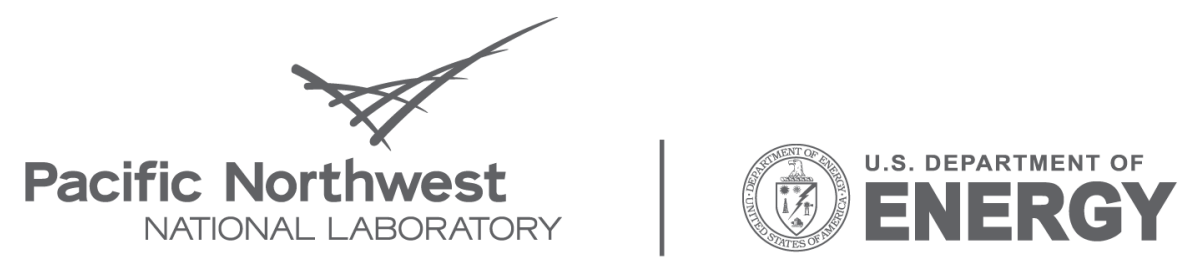

Proudly Operated by Battelle Since 1965

902 Battelle Boulevard

P.O. Box 999

Richland, WA 99352

1-888-375-PNNL (7665)

www.pnl.gov 